\title{
EVALUACIÓN AGRONÓMICA Y NUTRICIONAL DEL PASTO ESTRELLA AFRICANA (Cynodon nlemfuensis) EN LA ZONA DE MONTEVERDE, PUNTARENAS, COSTA RICA. II. VALOR NUTRICIONAL
}

\author{
Luis Villalobos ${ }^{1 / *}$, Jose Arce
}

\begin{abstract}
Palabras clave: Valor nutricional, manejo pastoreo, pastos tropicales, Cynodon nlemfuensis. Keywords: Nutritional value, grazing management, tropical grasses, Cynodon nlemfuensis.
\end{abstract}

Recibido: 09/09/13

\section{RESUMEN}

Se analizó el valor nutricional del pasto estrella africana (Cynodon nlemfuensis) a lo largo de 2 años en muestreos bimensuales, en 4 fincas comerciales de ganado lechero ubicadas en los cantones de Tilarán y Central (latitud $10^{\circ} 20^{\prime}$ $\mathrm{N}$, longitud $84^{\circ} 50^{\prime}$, altitud 800 a $1200 \mathrm{msnm}$ ) de las provincias de Guanacaste y Puntarenas, respectivamente. Las muestras se recolectaron en el aparto siguiente a ser pastoreado y se utilizó una altura de cosecha de $10 \mathrm{~cm}$, para simular el pastoreo que hacen los animales. La composición nutricional promedio para los 2 años de evaluación fue de 23,57\% MS, 20,27\% PC, 2,67\% EE, $10,97 \%$ cenizas, $64,21 \%$ FDN, 34,95\% FDA, $4,06 \%$ lignina y $68,02 \%$ DIVMS y su contenido energético para las variables de TND, ED, EM, $\mathrm{EN}_{\mathrm{L}}(3 \mathrm{X})$ y $\mathrm{EN}_{\mathrm{G}}$ fue $61,37 \% ; 2,71 ; 2,05 ; 1,25 \mathrm{y}$ 0,78 Mcal. $\mathrm{kg}^{-1}$ de MS, respectivamente. El valor nutricional del pasto estrella africana varió a lo largo del año como resultado de la climatología de la zona de Monteverde, siendo las fincas con influencia de la vertiente del Pacífico las de menor afectación en la calidad del forraje. El pasto estrella africana mostró un contenido de PC superior a lo reportado para dicha especie $\mathrm{y}$, en general, para pastos tropicales, por lo cual

1 Autor para correspondencia. Correo electrónico: luis.villalobosvillalobos@ucr.ac.cr
Aceptado: 12/02/14

\begin{abstract}
Agronomic and nutritional evaluation of african star grass-based pastures (Cynodon nlemfuensis) in the region of Monteverde, Puntarenas, Costa Rica. II. Nutritional Value. The nutritional value of african star grass (Cynodon nlemfuensis)-based pastures was analyzed in 4 commercial dairy farms in the Monteverde region (latitude $10^{\circ} 20^{\prime}$, longitude $84^{\circ} 50^{\prime}, 800$ to 1200 masl), every other month during a 2-year period. Sampling was carried out in the paddock ready to be grazed and samples were hand-plucked leaving a $10 \mathrm{~cm}$ stubble height, simulating the consumption made by lactating cows. Average nutritional value for the 2 years was $23.57 \% \mathrm{DM}, 20.27 \% \mathrm{CP}, 2.67 \%$ EE, $10.97 \%$ ashes, $64.21 \%$ NDF, 34.95\% ADF, $4.06 \%$ lignin and $68.02 \%$ IVDMD on a dry matter basis. Estimated energy content for the variables of TDN, DE, ME, $\mathrm{NE}_{\mathrm{L}}(3 \mathrm{X})$, and $\mathrm{NE}_{\mathrm{G}}$ was $61.37 \%, 2.71,2.05,1.25$ and 0.78 Mcal.kgDM, respectively. Nutritional value of african star grass varied along the year, as it is sensitive to weather conditions. The farms with influence from the Pacific slope are less affected in forage quality than those with Atlantic influence. CP content was higher in this study than in
\end{abstract}

\footnotetext{
* Centro de Investigaciones en Nutrición Animal y Escuela de Zootecnia, Universidad de Costa Rica. San José, Costa Rica.
} 
no es limitante para la producción láctea, y la suplementación del ganado lechero en la zona debe utilizar fuentes que permitan una utilización eficiente del $\mathrm{N}$ soluble a nivel ruminal. La rotación del pasto estrella cada 25 días debe ser flexible para permitir, en conjunto con programas de fertilización, optimizar la productividad de las pasturas y su persistencia.

\section{INTRODUCCIÓN}

Los pastos tropicales se caracterizan por su valor nutricional medio a bajo debido a su contenido proteico típicamente bajo y una proporción de pared celular alta que limitan la producción de proteína microbiana en el rumen (Pérez et ál. 2001, Villarreal et ál. 2006). Diferentes especies y cultivares mejorados han aportado un grado de avance significativo debido a su producción de biomasa y valor nutricional mayores que les confieren un potencial mayor para condiciones de trópico (Dzowela et ál. 1990). Entre las estrategias de mayor difusión para el mejoramiento de la producción animal en sistemas de pastoreo, se menciona la adopción de leguminosas y el mejoramiento de la calidad nutritiva de las gramíneas mediante la selección de accesiones superiores o programas de hibridación (Lascano 2002).

Las gramíneas tropicales como el estrella africana y el bermuda son altamente sensibles a cambios en las horas luz durante el año, afectadas tanto en la producción de biomasa como el valor nutricional (Sinclair et ál. 2001, Hill et ál. 1993, Dzowela et ál. 1990); asimismo el género Cynodon se caracteriza por su capacidad de extraer sustanciales cantidades de nutrientes del suelo (Pant et ál. 2004). El pasto estrella africana debe tener un período de recuperación entre 4 a 5 semanas entre pastoreos sucesivos de tal forma que su persistencia no se vea afectada para mantener una producción de materia seca alta (12 a 17 previous evaluations for this species elsewhere and, in general, for tropical grasses as well. Protein is not a limiting factor for dairy cattle grazing african star grass, and its supply must use sources that allow an efficient utilization of soluble $\mathrm{N}$ by rumen microorganisms. The 25 -day rotation systems in Monteverde must be flexible to allow, jointly with fertilization programs, the optimization of the productivity and persistence of the pastures.

ton.ha $\left.{ }^{-1}\right)$, y contenido proteico (11 a $16 \%$ ) y digestibilidad (55 a 60\%) adecuadas (Mislevy 2002). $\mathrm{Si}$ bien es cierto bajo condiciones tropicales no hay una estacionalidad marcada como en climas templados, la producción de biomasa varía durante el año y, por tanto, el manejo de las edades de cosecha debe garantizar un valor nutricional óptimo para los animales en producción (Pant et ál. 2004, Mandebvu et ál. 1999, Sánchez y Soto 1996) que se ajuste a la capacidad de carga de las explotaciones (Hill et ál. 1993) sin sacrificar la ganancia de peso o la producción láctea (Dzowela et ál. 1990).

La suplementación en pasturas tropicales debe satisfacer los nutrientes limitantes en los forrajes y optimizar su utilización en el rumen por medio de un suministro constante para los animales en crecimiento o en producción. Mediante un consumo adecuado de nutrientes los microrganismos del rumen pueden reproducirse mientras se estimula la tasa de pasaje y el consumo voluntario (Ortiz et ál. 2002). La cantidad de nitrógeno disponible para la síntesis de proteína microbial puede incrementarse en pastos tropicales, hasta cierto punto mediante el incremento de las dosis de fertilización nitrogenada (Johnson et ál. 2001). El manejo de una adecuada suplementación reporta beneficios no solo en producción animal mediante un consumo de materia seca mayor (Villarreal et ál. 2006) sino también en el medio ambiente ya que dietas ricas en fibra producen más acetato a nivel ruminal mientras 
que en dietas con alto contenido de carbohidratos solubles o fácilmente degradables predomina la producción de propionato, lo cual puede reducir la producción de metano (Lascano 2002).

El objetivo de la presente investigación fue estimar el valor nutricional del pasto estrella africana y sus variaciones en 4 fincas dedicadas a la producción láctea en la zona de Monteverde, a lo largo de 2 años.

\section{MATERIALES Y MÉTODOS}

En Villalobos y Arce (2013) se describe la zona, las fincas evaluadas y las características climáticas de la zona donde se realizó la presente investigación cuya duración fue de 2 años (febrero 2007 a marzo 2009). Se tomó un total de 48 muestras (1 muestra por finca por muestreo bimensual) de forma aleatoria para estimar el valor nutricional del pasto estrella africana con una altura de cosecha de $10 \mathrm{~cm}$ sobre el suelo, para simular el pastoreo que realizan los animales. Se determinó el contenido de materia seca (MS) y proteína cruda (PC) (AOAC 1990), extracto etéreo (EE) y cenizas (AOAC 2000), las fracciones de la pared celular (FDN, FDA y lignina) (Van Soest y Robertson 1985), y la digestibilidad "in vitro" de la materia seca (DIVMS) (Van Soest et ál. 1991). La energía en sus diferentes expresiones (TND, ED, EM, $\mathrm{EN}_{\mathrm{L}} 3 \mathrm{X}$ y $\mathrm{EN}_{\mathrm{G}}$ ) se estimó mediante las metodologías descritas en el NRC (2001).

La información obtenida para las variables de valor nutricional, excepto lignina y DIVMS, fue sometida a un modelo lineal generalizado mediante el procedimiento PROC GLM del paquete estadístico SAS/STAT® 9.3:

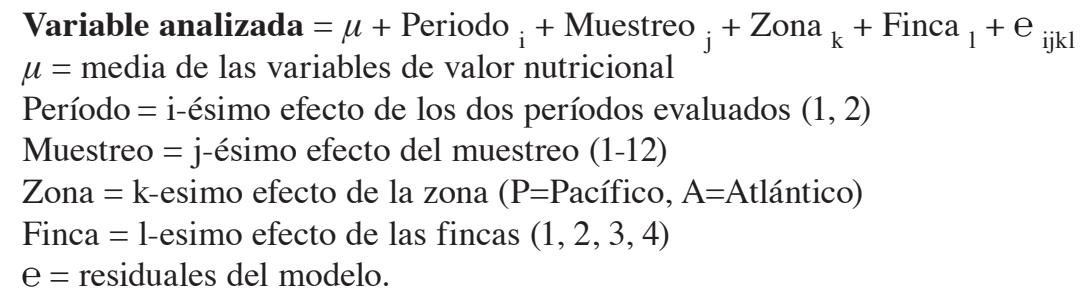

Se utilizó un modelo lineal general reducido mediante el procedimiento PROC GLM del paquete estadístico SAS/STAT ${ }^{\circledR}$ 9.3. Para dicho modelo se consideró únicamente las zonas y fincas para el análisis de lignina y DIVMS. Se aplicó la prueba de Duncan para establecer diferencias significativas $(p \leq 0,05)$ entre medias en los diferentes períodos, muestreos, zonas y fincas para todas las variables. Ambos modelos buscaron efectos significativos $(\mathrm{p} \leq 0,05)$ en base al error tipo I.

\section{RESULTADOS Y DISCUSIÓN}

\section{Componentes intracelulares}

El contenido de MS promedio del pasto estrella africana fue de $23,57 \%$ y mostró ser altamente significativo $(\mathrm{p}<0,0001)$ para el modelo utilizado y entre los diferentes muestreos ( $\mathrm{p}<0,0001)$ (Cuadro 1). Dichos resultados coinciden con lo encontrado por Salazar (2007) y dicho valor es similar al reportado por Sánchez y Soto (1999a) con el pasto estrella africana en los distritos de Quesada y Fortuna del cantón de San Carlos, respectivamente. No se encontró diferencias significativas $(p<0,05)$ en el contenido de MS del pasto estrella entre las fincas, períodos y zonas evaluadas. El contenido de MS del pasto estrella africana varió con base en la climatología en la región de Monteverde, que mostró valores de hasta $29,47 \%$ en época seca hasta llegar a $18,55 \%$ en época lluviosa (Figura 1); dichos valores mostraron ser superiores a los encontrados por Salazar (2007) y una tendencia similar fue reportada por Sánchez y Soto (1999a) con pastos 
Cuadro 1. Contenido de materia seca, proteína cruda, extracto etéreo y cenizas del pasto estrella africana en 4 fincas de ganado lechero evaluadas durante 2 años, en Monteverde, Puntarenas.

\begin{tabular}{|c|c|c|c|c|c|}
\hline Finca & Zona & $\begin{array}{c}\text { Materia seca } \\
(\%)\end{array}$ & $\begin{array}{c}\text { Proteína cruda } \\
(\%)\end{array}$ & $\begin{array}{c}\text { Extracto etéreo } \\
(\%)\end{array}$ & $\begin{array}{c}\text { Cenizas } \\
(\%)\end{array}$ \\
\hline 1 & $\mathrm{P}$ & 23,36 & 20,68 & 2,84 & $10,72^{\mathrm{b}}$ \\
\hline 2 & $\mathrm{P}$ & 24,05 & 20,44 & 2,31 & $10,95^{\mathrm{ab}}$ \\
\hline 3 & A & 23,57 & 19,79 & 2,58 & $10,94^{\mathrm{ab}}$ \\
\hline 4 & A & 23,32 & 20,17 & 2,96 & $11,27^{\mathrm{a}}$ \\
\hline Promedio $^{1}$ & & 23,57 & 20,27 & 2,67 & 10,97 \\
\hline Variable del modelo & & \multicolumn{4}{|c|}{ Significancia estadística $(\mathrm{P})$} \\
\hline Período & & $\mathrm{ns}^{2}$ & $\mathrm{~ns}$ & ns & 0,0325 \\
\hline Muestreo & & $<0,0001$ & $<0,0001$ & $\mathrm{~ns}$ & $<0,0001$ \\
\hline Zona & & ns & ns & ns & ns \\
\hline Finca & & ns & ns & ns & ns \\
\hline
\end{tabular}

a, b, medias con letras diferentes dentro de una misma columna difieren entre sí, según la prueba de Duncan $(\mathrm{p} \leq 0,05)$

1. Los valores corresponden al promedio de 48 muestras. 2. No significativo.

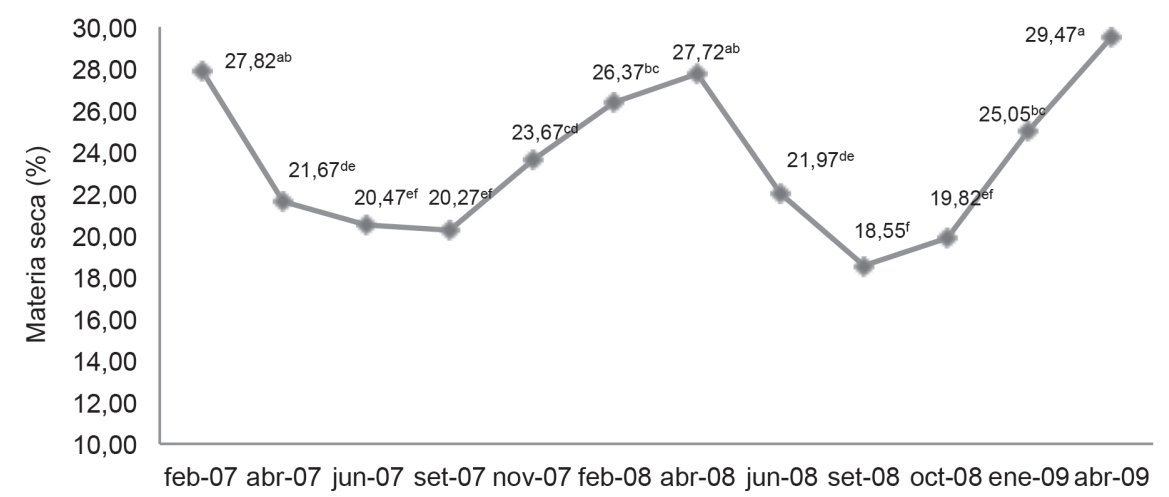

Fig. 1. Contenido de materia seca del pasto estrella africana a lo largo de 2 años en Monteverde, Puntarenas (a, b, c medias con letras diferentes difieren entre sí, según la prueba de Duncan $(\mathrm{p} \leq 0,05))$.

de piso tropicales. Las fincas de Monteverde con influencia de la región Pacífico son afectadas más directamente en época seca (Retana 2012, Hill et ál. 1993). Debido a las variaciones en el contenido de la MS del pasto estrella africana en la zona de Monteverde, los productores de ganado de leche deben monitorear y ajustar las dietas de sus hatos con el fin de asegurar un consumo de MS y de fibra efectiva proveniente del pasto, principalmente en los meses de transición (inicio de época seca e inicio de época lluviosa), lo cual finalmente puede afectar el contenido de sólidos en leche y el pago al productor (Cruz y Sánchez 2000). Debido a que la mayoría de productores mantienen una carga animal constante durante el año en la zona de Monteverde, podría haber 
variaciones en el consumo de MS del pasto, que afecta la productividad láctea y aumento del riesgo de condiciones metabólicas como la acidosis, que pueden prevenirse al aumentar el consumo de materia seca de pasto o alguna fuente fibrosa que promueva la rumia y la salivación, como por ejemplo mediante el uso de paca de heno en ciertas épocas del año.

El contenido de PC promedio del pasto estrella africana fue de $20,27 \%$ y fue altamente significativo con base en el modelo utilizado $(\mathrm{p}<0,0001)$ y entre los diferentes muestreos $(\mathrm{p}<0,0001)$ (Figura 2); lo anterior fue reportado por Salazar (2007) para la zona de San Carlos con pasto estrella africana. No hubo diferencias significativas $(p<0,05)$ entre las fincas, zonas y períodos evaluados en el contenido de PC. La PC del pasto estrella varió con base en la climatología de la región de Monteverde que a su vez, afecta la fenología de la planta al mostrar valores de hasta 25,58\% en los meses de mayor precipitación (setiembre a noviembre) donde se evidenció de forma consistente entre fincas un número de hojas por rebrote promedio de 2,87 (Villalobos y Arce 2013). En los meses de época seca (enero a abril) el contenido de PC bajó hasta 16,07\%; dicho comportamiento ha sido reportado previamente para el pasto estrella pues la presencia de menor humedad en el suelo limita la movilidad del nitrógeno hacia y dentro de la planta (Pérez et ál. 2001, Sánchez y Soto 1996). Hill et ál. (1993) encontraron en 2 cultivares de pasto bermuda que luego de períodos en los que la tasa fotosintética se ve reducida (invierno en sub-trópico o verano en trópico), las plantas pueden agotar rápidamente el $\mathrm{N}$ disponible en el suelo al disponer de humedad para movilizar los nutrientes, razón por la cual la fertilización al inicio de la época lluviosa no debe limitar la capacidad de desarrollo vegetativo de las plantas. Johnson et ál. (2001) evaluaron el efecto de la fertilización nitrogenada sobre el contenido de PC en pasto estrella, bermuda (Cynodon dactylon) y bahía (Paspalum notatum); al ser su contenido menor durante la época seca, sin embargo el primero mostró de forma consistente un contenido mayor entre muestreos y dosis aplicadas. Pant et ál. (2004) evaluaron el valor nutricional del pasto estrella africana durante 3 años bajo diferentes programas de fertilización y encontraron un rango en el contenido de PC entre 8,7 a $28,3 \%$; las dosis menores a $23 \mathrm{~kg} \cdot \mathrm{ha}^{-1} \mathrm{de}$ potasio $(\mathrm{K})$ mostraron contenidos de PC mayores, en la zona de Monteverde los programas de fertilización reportados de las fincas evaluadas fueron de $10 \mathrm{~kg} \cdot \mathrm{ha}^{-1}$ de $\mathrm{K}$ anuales en aplicaciones fraccionadas (Villalobos y Arce 2013).

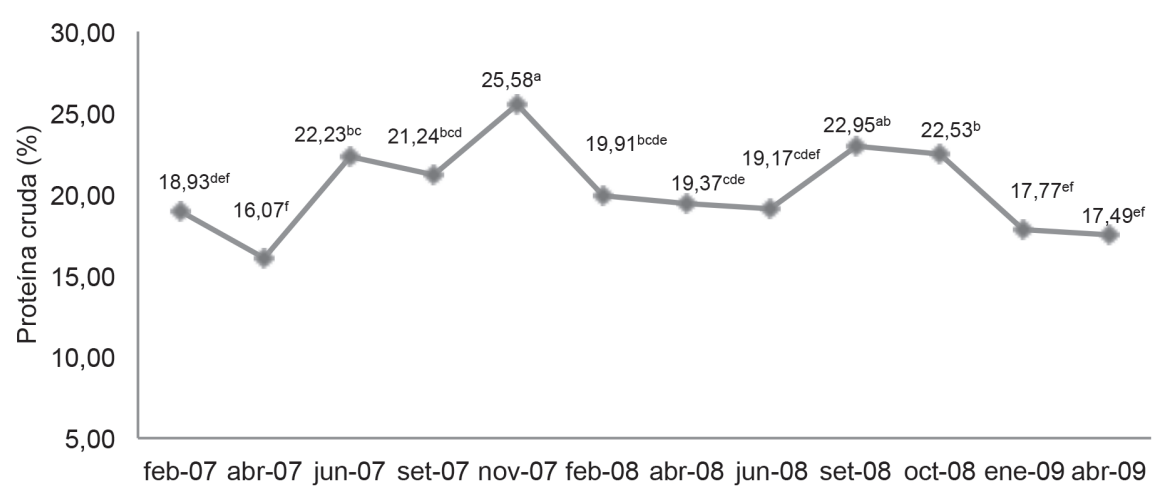

Fig. 2. Contenido de proteína cruda del pasto estrella africana a lo largo de 2 años en Monteverde, Puntarenas (a, b, c medias con letras diferentes difieren entre sí, según la prueba de Duncan $(\mathrm{p} \leq 0,05))$. 
El aumento en el contenido proteico de los pastos no va a reportar necesariamente mayores ganancias en producción animal (Hill et ál. 1993) sino que incluso puede resultar negativo (Lascano 2002). Es por esto, que las variaciones en el contenido de PC deben considerarse dentro de la suplementación con el objetivo de disminuir el riesgo de cambios bruscos en la producción (Mandebvu et ál. 1999). Sánchez y Soto (1999a) no encontraron diferencias importantes entre épocas climáticas en pastos de piso y de corte por lo cual recomiendan que el alimento balanceado utilizado tenga el mismo nivel de PC a lo largo del año; dicha situación tiene mayor complejidad para la región de Monteverde pues sí se presentaron diferencias de gran magnitud (hasta 10 unidades porcentuales) en el contenido de PC en el pasto durante el año. Mediante el ajuste de las raciones del ganado lechero, se puede optimizar la utilización de la proteína soluble en rumen al brindar fuentes de carbohidratos solubles que contribuyen en la producción de proteína microbial y disminuyen la absorción excesiva de amonio ruminal hacia el torrente sanguíneo (Elizalde et ál. 1999). Asimismo se debe considerar las condiciones en los apartos para garantizar un adecuado consumo de forraje principalmente en época lluviosa en donde el acceso de los animales al pasto puede verse limitado y afectar las características físicas del suelo (WingChing et ál. 2009). La producción del pasto estrella africana en Monteverde, a diferencia de otras latitudes tropicales, no presenta una estacionalidad marcada, por lo que incluso en época seca se encontró un contenido de PC adecuado para animales en producción (Ortiz et ál. 2002, Pérez et ál. 2001). El manejo de la fertilización ajustada a las condiciones climatológicas permite que la utilización de los nutrientes disponibles en el suelo sea más eficiente y por tanto una mayor eficiencia en el flujo de nutrientes hacia la producción láctea.

En un trabajo previo (Villalobos 2012), se analizó el fraccionamiento proteico del pasto estrella africana en fincas de Monteverde y se encontró que la fracción soluble es mayor a la de los pastos ryegrass perenne (Lolium perenne) y alpiste forrajero (Phalaris arundinacea). En dosis de fertilización superiores a $157 \mathrm{~kg} \cdot \mathrm{ha}^{-1} \cdot \mathrm{año}^{-1} \mathrm{de}$ $\mathrm{N}$ Johnson et ál. (2001) reportan que un $42,1 \%$ del $\mathrm{N}$ en la planta se encuentra en la fracción A o soluble, valor cercano al encontrado en la zona de Monteverde $(38,52 \%)$ cuyas dosis fueron superiores $\left(250 \mathrm{~kg} \cdot \mathrm{ha}^{-1}\right.$ de N) lo cual puede deberse a que la producción de pasto se da durante todo el año a diferencia de lo reportado por dichos autores. Los suplementos brindados al ganado lechero en fincas de Monteverde deben proveer fuentes energéticas fácilmente degradables en el rumen que favorezcan la producción de microrganismos al promover un mayor consumo del $\mathrm{N}$ amoniacal y una tasa de digestión de la ración total mayor (Lascano 2002, Alayon et ál. 1998, Villarreal et ál. 2006, Sánchez y Soto 1999a). Pérez et ál. (2001) reportaron contenidos de PC en el pasto estrella entre 5,2 a 7,9\%, dichos valores se consideran limitantes en proteína; mientras que en la leguminosa kudzú (Pueraria phaseoloides) los mismos autores reportaron un $16 \%$ de PC, dicho valor es similar a los del pasto estrella en época seca en Monteverde; evidencia de que la zona de Monteverde presenta condiciones favorables para la producción láctea pues el contenido de $\mathrm{PC}$ no bajó a valores limitantes. La inclusión de leguminosas como el madero negro (Gliricidia sepium) en una dieta basal de heno de pasto estrella africana limitante en PC, ha reportado incrementos en el consumo de MS, materia orgánica y PC; según lo encontrado en el presente estudio el pasto estrella africana no presenta limitantes de nitrógeno para la producción de microrganismos a nivel ruminal (Alayon et ál. 1998). El aumento en PC que se encontró en ciertas épocas del año en el pasto estrella africana, podría predisponer a desbalances en el consumo de nutrientes pues el manejo de las raciones se mantiene relativamente constante. Por ello es importante en este sentido tomar en cuenta que se debe sincronizar el aporte de carbohidratos no fibrosos y proteína soluble en el rumen con una relación 3:1 de manera que se optimice la producción de proteína microbial (Arroyo et ál. 2003). En la presente investigación se estimó el contenido promedio de carbohidratos 
no fibrosos y PC para el pasto estrella en 1,88\% y $20,27 \%$, respectivamente. Por lo que si se parte del hecho de que el $38,52 \%$ de esa proteína es soluble (Villalobos 2012), se obtiene una relación carbohidratos no fibrosos: proteína soluble equivalente a 0,24:1 lo cual indica la necesidad de suplementar con fuentes altas en carbohidratos fácilmente disponibles como la melaza que permitan optimizar la utilización del pasto estrella en el rumen, y prestar especial atención en las épocas del año en que el contenido de PC del pasto es mayor.

El contenido de EE promedio del pasto estrella africana fue de $2,67 \%$ y no mostró ser significativo $(p<0,05)$ con base en el modelo utilizado ni tampoco para los períodos, fincas, muestreos y zonas evaluados (Cuadro 1). El EE fue superior al reportado por Salazar (2007) y similar a lo encontrado por Sánchez y Soto (1996) para el mismo pasto en la zona de San Carlos con 0,91\% y 2,12 a $2,33 \%$, respectivamente. De cualquier manera el bajo contenido de grasa presente en el pasto, abre la posibilidad de suplementar con subproductos de mayor contenido graso y/o con grasas protegidas como complemento al pastoreo de estrella africana en niveles prudentes que no interfieran con la digestión de la fibra del pasto (NRC 2001) que permitan incrementar la densidad energética de la ración con efectos positivos en producción de leche, pues el hecho de ser un pasto de buena calidad nutricional y alto contenido de PC favorece las condiciones adecuadas para mejorar de manera eficiente la producción láctea mediante la utilización de jabones del calcio (Campabadal 1996).

El contenido de cenizas promedio para el pasto estrella fue de $10,97 \%$ y fue altamente significativo con base en el modelo evaluado y los muestreos $(\mathrm{p}<0,0001)$ lo cual puede deberse a la variación que se da en la absorción de minerales por la planta, así como la movilidad de los mismos en respuesta a la cantidad de agua presente en el suelo. El contenido de cenizas fue significativo con base en los 2 períodos $(\mathrm{p}<0,0325)$ incluidos en el presente estudio. Dicho contenido es superior al encontrado por Salazar (2007) y
Sánchez y Soto (1996) con 8,69\% y 9,0\%, respectivamente, en fincas de la zona de San Carlos.

\section{Componentes de la pared celular}

El contenido de pared celular (FDN) promedio del pasto estrella africana fue $64,21 \%$; que muestra una alta significancia para el modelo utilizado y entre los diferentes muestreos $(\mathrm{p}<0,0001)$ y a su vez fue significativo entre los períodos de evaluación $(\mathrm{p}<0,0004)$. El contenido de FDN fue menor al reportado por varios autores para el pasto estrella africana (Salazar 2007, Sánchez y Soto 1999a, Johnson et ál. 2001, Hill et ál. 1993), con valores entre 71,1 a 75,5\%. Pérez et ál. (2001) reportaron un contenido de FDN de 76,3\% en un sistema de pastoreo con acceso a bancos de proteína de kudzú durante el día e indican que un contenido de pared celular mayor repercute negativamente en la digestibilidad de la MS, lo que origina a su vez una producción animal baja; los resultados de la presente investigación indican que se podrían esperar consumos de MS mayores debido al contenido de FDN menor con respecto a otros autores. El pasto estrella africana en la zona de Monteverde mostró cambios en el contenido de FDN durante el año, al presentar valores mayores en época seca y menores en época lluviosa (Figura 3); dicho comportamiento ha sido reportado en condiciones tropicales (Salazar 2007, Andrade 2006, Villalobos y Sánchez 2010) y debe ser tomado en cuenta para hacer ajustes en las raciones que prevengan problemas de acidosis por falta de fibra efectiva o por el contrario problemas de consumo de materia seca por excesos de fibra en la ración (Allen 2000, Cruz y Sánchez 2000). Además, se sabe que la edad de cosecha de los pastos del género Cynodon influye sobre la proporción de las fracciones de hemicelulosa, celulosa y lignina dentro de la FDN, al ser menor la primera y al aumentar las otras 2 conforme se prolonga los días de cosecha (Mandebvu et ál. 1999), lo cual puede verse afectado por el manejo particular que se le dé al pasto en cada finca. 


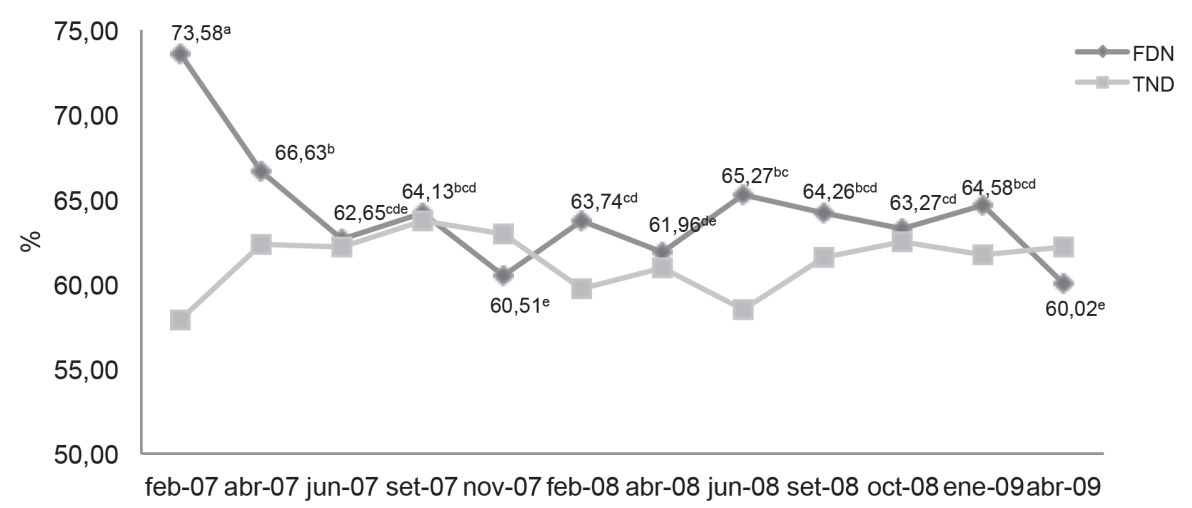

Fig. 3. Contenido de pared celular y total de nutrientes digestibles del pasto estrella africana a lo largo de 2 años en Monteverde, Puntarenas.

El contenido de FDA promedio fue $34,95 \%$ y mostró significancia entre los diferentes muestreos $(\mathrm{p}<0,0274)$ (Cuadro 2). El contenido de FDA fue, al igual que la FDN, inferior al encontrado por Salazar (2007) en el pasto estrella africana en la zona de San Calos con 37,88\% y cuyo comportamiento varió significativamente $(\mathrm{p}<0,05)$ entre los meses del año. Johnson et ál. (2001) reportaron contenidos de FDA entre 30,9 a 33,9\% para el pasto estrella africana, quienes mencionan que la fertilización de $\mathrm{N}$ tiene efectos mínimos sobre dicha variable. El contenido de lignina promedio del pasto estrella africana en Monteverde fue de 4,06\%; dicha variable no mostró significancia con base en el modelo utilizado (reducido), asimismo no hubo diferencias entre las zonas ni fincas evaluadas (Cuadro 2). El contenido de lignina del pasto estrella africana fue menor al encontrado por Salazar (2007) con $5,65 \%$ y mostró una tendencia similar al no

Cuadro 2. Contenido de los componentes de la pared celular del pasto estrella africana en 4 fincas de ganado lechero evaluadas durante 2 años, en Monteverde, Puntarenas.

\begin{tabular}{lcccc}
\hline Finca & Zona & FDN $^{1}(\%)$ & FDA $^{2}(\%)$ & Lignina $(\%)$ \\
\hline 1 & $\mathrm{P}$ & 63,95 & 35,04 & 3,99 \\
2 & $\mathrm{P}$ & 64,31 & 34,52 & 4,32 \\
3 & $\mathrm{~A}$ & 64,55 & 35,09 & 4,07 \\
4 & $\mathrm{~A}$ & 64,05 & 35,17 & 3,88 \\
\hline Promedio $^{3}$ & & 64,21 & 34,95 & 4,06 \\
\hline Variable del modelo & & Significancia estadística $(\mathrm{P})$ & --- \\
\hline Período & & $\mathrm{ns}$ & --- \\
Muestreo & 0,0004 & 0,0274 & $\mathrm{~ns}$ \\
Zona & $<0,0001$ & $\mathrm{~ns}$ & $\mathrm{~ns}$ \\
Finca & & $\mathrm{ns}$ & $\mathrm{ns}$ & \\
\hline
\end{tabular}

$\mathrm{a}, \mathrm{b}$, medias con letras diferentes dentro de una misma columna difieren entre sí, según la prueba de Duncan ( $\mathrm{p} \leq 0,05)$.

1. Fibra detergente neutro. 2. Fibra detergente ácida. 3. Los valores corresponden al promedio de 48 muestras. 4. No significativo. 
mostrar diferencias significativas $(\mathrm{p}<0,05)$ entre las fincas evaluadas. El proceso de lignificación en pastos tropicales reduce la degradación de la hemicelulosa y la celulosa en el rumen y en consecuencia hay menos energía disponible para el animal (Lascano 2002). La FDA y la lignina presentaron valores menores a los reportados en literatura para el pasto estrella lo cual ofrece potencial para favorecer el consumo de MS proveniente del pasto en la zona de Monteverde, sin embargo al tomar como referencia los requerimientos nutricionales para ganado lechero y los parámetros ideales que debe tener la ración en términos de la fibra y sus componentes (Cruz y Sánchez 2000 y NRC 2001) se hace evidente la necesidad de suplementar las raciones con materiales de menor contenido de fibra de modo que los animales puedan consumir suficiente materia seca para cubrir sus requerimientos nutricionales sin experimentar un llenado físico que lo impida.

\section{Digestibilidad in vitro de la materia seca (DIVMS) y contenido de energía estimada}

La DIVMS promedio del pasto estrella africana fue de $68,02 \%$ y no fue significativa $(\mathrm{p}<0,05)$ con base en el modelo, zona y fincas analizados; sin embargo el rango de dichos valores comprendió entre 63 a $74 \%$ en las fincas evaluadas, con medias de $69,29 \%$ y $66,75 \%$ para las fincas con influencia Pacífico y Atlántico, respectivamente (Cuadro 3). El valor encontrado fue similar al mencionado por Salazar (2007) y Sánchez y Soto (1999a) con 68,52\% y 70,2\% en la región de San Carlos, y superior al reportado por Pérez et ál. (2001) y Pant et ál. (2004) con $40,8 \%$ y entre 35 a $60 \%$, respectivamente. Lascano (2002) menciona que aumentos relativamente pequeños en la digestibilidad de una gramínea han sido utilizados en la selección de variedades en los últimos 30 años y pueden tener un gran impacto en la producción animal como ha sido

Cuadro 3. Digestibilidad in vitro y contenido de energía estimada del pasto estrella africana en 4 fincas de ganado lechero evaluadas durante 2 años, en Monteverde, Puntarenas.

\begin{tabular}{|c|c|c|c|c|c|c|c|}
\hline Finca & Zona & $\begin{array}{c}\text { DIVMS }^{1} \\
(\%)\end{array}$ & $\begin{array}{l}\mathrm{TND}^{2} \\
(\%)\end{array}$ & $\begin{array}{c}\mathrm{ED}^{3} \\
\text { (Mcal.kg-1 MS) }\end{array}$ & $\begin{array}{c}\mathrm{EM}^{4} \\
\left(\mathrm{Mcal} . \mathrm{kg}^{-1} \mathrm{MS}\right)\end{array}$ & $\begin{array}{c}\mathrm{EN}_{\mathrm{L}} 3 \mathrm{X}^{5} \\
\left(\mathrm{Mcal} . \mathrm{kg}^{-1} \mathrm{MS}\right)\end{array}$ & $\begin{array}{c}\mathrm{EN}_{\mathrm{G}}{ }^{6} \\
\left(\mathrm{Mcal} . \mathrm{kg}^{-1} \mathrm{MS}\right)\end{array}$ \\
\hline 1 & $\mathrm{P}$ & 71,79 & $63,07^{\mathrm{a}}$ & $2,78^{\mathrm{a}}$ & $2,11^{\mathrm{a}}$ & $1,29^{\mathrm{a}}$ & $0,83^{\mathrm{a}}$ \\
\hline 2 & $\mathrm{P}$ & 66,79 & $62,72^{\mathrm{a}}$ & $2,77^{\mathrm{a}}$ & $2,09^{\mathrm{a}}$ & $1,28^{\mathrm{a}}$ & $0,82^{\mathrm{a}}$ \\
\hline 3 & A & 66,27 & $59,45^{\mathrm{b}}$ & $2,63^{\mathrm{b}}$ & $1,97^{\mathrm{b}}$ & $1,20^{\mathrm{b}}$ & $0,73^{\mathrm{b}}$ \\
\hline 4 & A & 67,23 & $60,22^{\mathrm{b}}$ & $2,67^{\mathrm{b}}$ & $2,00^{\mathrm{b}}$ & $1,23^{\mathrm{b}}$ & $0,75^{\mathrm{b}}$ \\
\hline Promedio $^{7}$ & & 68,02 & 61,37 & 2,71 & 2,05 & 1,25 & 0,78 \\
\hline \multicolumn{3}{|c|}{ Variable del modelo } & \multicolumn{5}{|c|}{ Significancia estadística (P) } \\
\hline Período & & --- & ns & ns & ns & ns & ns \\
\hline Muestreo & & --- & $<0,0001$ & $<0,0001$ & $<0,0001$ & 0,0043 & $<0,0001$ \\
\hline Zona & & $\mathrm{ns}$ & $<0,0001$ & $<0,0001$ & $<0,0001$ & $<0,0001$ & $<0,0001$ \\
\hline Finca & & ns & $\mathrm{ns}$ & ns & ns & ns & ns \\
\hline
\end{tabular}

$\mathrm{a}, \mathrm{b}$, medias con letras diferentes dentro de una misma columna difieren entre sí, según la prueba de Duncan ( $\mathrm{p} \leq 0,05$ ).

1. Digestibilidad in vitro de la materia seca. 2. Total de nutrientes digestibles. 3. Energía digestible. 4. Energía metabolizable.

5. Energía neta de lactancia. 6. Energía neta de ganancia. 7. Los valores corresponden al promedio de 48 muestras. 
demostrado por las correlaciones altas mostradas entre la DIVMS con las ganancias de peso (Dzowela et ál. 1990). Mandebvu et ál. (1999) mencionan que la digestibilidad en 2 cultivares de pasto bermuda (Tifton 85 y Coastal), disminuyó al aumentar la edad de cosecha de 3 a 8 semanas de 62,1 a $51,0 \%$. La fertilización con $\mathrm{P}$ y K permite producción de forraje de pasto estrella de valor nutricional mayor; el efecto del $\mathrm{P}$ sin embargo puede volverse menos evidente conforme pasa el tiempo debido a que la planta inicialmente dispone del mismo para producción de biomasa radicular que posteriormente es renovada con requerimientos menores a los iniciales, es fundamental conocer la capacidad productiva del pasto estrella a través de la fertilización con $\mathrm{N}$ para hacer una utilización eficiente de los nutrientes disponibles (Johnson et ál. 2001, Pant et ál. 2004).

El total de nutrimentos digestibles (TND) promedio fue de $61,37 \%$ y mostró una alta significancia $(p<0,0001)$ con base en el modelo estadístico utilizado, así como los muestreos y las zonas evaluadas (Cuadro 3). Dicho valor fue superior al encontrado por Salazar (2007) y Sánchez y Soto (1999b) con $51,15 \%$ y $53,8 \%$; esto puede deberse a que en el presente estudio el contenido de PC fue superior y los componentes de la pared celular fueron menores. La lignificación en pastos tropicales genera normalmente un desbalance entre energía y proteína en el rumen, lo cual afecta la digestión por los microrganismos al bloquear su contacto con polisacáridos de la pared celular, disminuyendo así el grado de degradabilidad y las fracciones potencialmente digestibles de la MS y la FDN (Lascano 2002, Mandebvu et ál. 1999). En el presente estudio se evidenció que el pasto estrella africana en la zona de Monteverde acumula menos lignina que lo reportado por Salazar (2007) lo cual puede explicar, en conjunto con su contenido proteico, el valor del TND superior encontrado con respecto a otras latitudes y cercano a pastos de clima templado como el ryegrass con $61,95 \%$ en la zona alta de Cartago (Villalobos y Sánchez 2010).

El contenido de TND mostró una relación inversa con respecto al contenido de FND durante los diferentes muestreos (Figura 3), al ser inferior en los meses de verano en donde el pasto estrella africana acumula más pared celular y superior en los meses de mayor precipitación en donde además de disminuir la FDN, la PC aumentó de forma significativa $(\mathrm{p}<0,05)$ (Figura 2). El mismo comportamiento fue encontrado por Salazar (2007) y Johnson et ál. (2001). La DIVMS y la digestibilidad de la FDN son indicadores del potencial productivo de los forrajes ya que el consumo voluntario se ve limitado por ambos componentes (Pérez et ál. 2001, Mandebvu et ál. 1999); lo cual indica que el pasto estrella tiene un potencial productivo mayor en la zona de Monteverde en comparación con otras latitudes.

Las fracciones energéticas estimadas de $\mathrm{ED}, \mathrm{EM}, \mathrm{EN}_{\mathrm{L}}$ y $\mathrm{EN}_{\mathrm{G}}$ mostraron valores promedio de 2,$71 ; 2,05 ; 1,25$ y 0,78 Mcal.kg-1 $^{-1}$ de MS, respectivamente (Cuadro 3). Las 4 fracciones de energía mostraron ser altamente significativas $(\mathrm{p}<0,0001)$ para el modelo, los muestreos y las zonas evaluadas. Las fracciones energéticas del pasto estrella africana en Monteverde mostraron ser superiores a las encontradas por Salazar (2007) y similares a las reportadas por Sánchez y Soto (1999b) en la zona de San Carlos para pasto estrella y pastos de piso tropicales, respectivamente. El período de recuperación de las pasturas de estrella africana en la zona de Monteverde permite obtener un forraje palatable de alto valor nutricional (Mislevy 2002). El contenido de $\mathrm{EN}_{\mathrm{L}}$ en el pasto estrella africana se ve afectado por la climatología (Figura 4), y presenta una mejora en el contenido energético del pasto al inicio de la época lluviosa durante los 2 años de evaluación, lo cual puede verse reflejado en un aumento en producción láctea o en mayores ganancias de peso (Dzowela et ál. 1990). Durante los meses de mayor precipitación, el pasto estrella mantuvo un valor nutricional superior con respecto a la época seca, dicho efecto fue más evidente en las fincas con influencia climatológica del Pacífico mientras que hacia la región Atlántica se presenta saturación de agua en los suelos de forma más frecuente lo cual disminuye la productividad y el valor nutricional de las pasturas que finalmente 


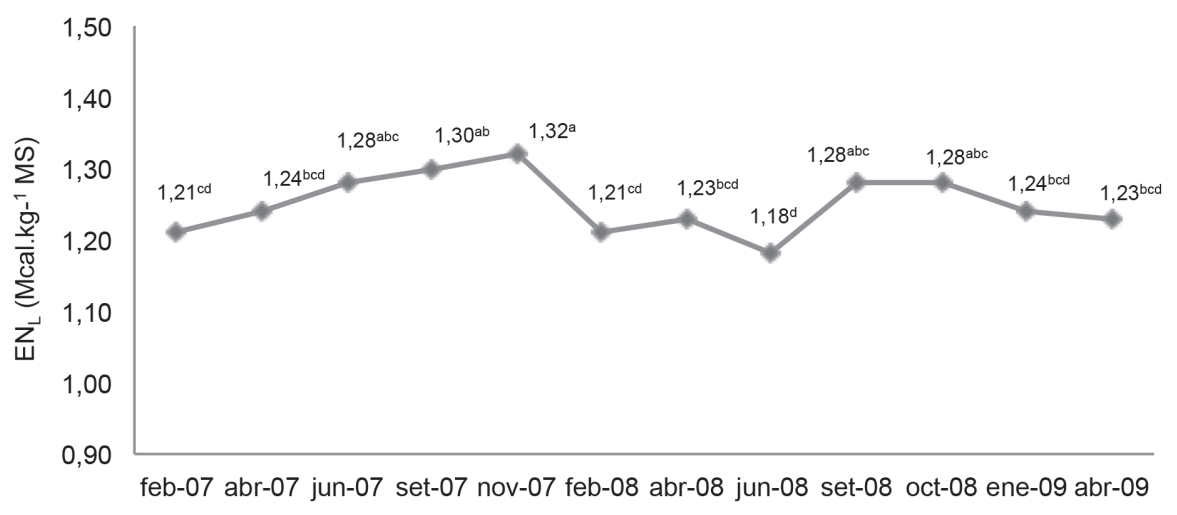

Fig. 4. Contenido de energía neta de lactancia del pasto estrella africana a lo largo de 2 años en Monteverde, Puntarenas. (a, b, c medias con letras diferentes difieren entre sí, según la prueba de Duncan $(\mathrm{p} \leq 0,05))$.

repercute en la producción láctea (WingChing et ál. 2008). Las variables climatológicas afectan el valor nutricional de los pastos e influyen sobre la curva de producción de leche durante el año, tal como ha sido evidenciado en la zona alta del Valle Central de Costa Rica (González y Boschini 1996). La suplementación con fuentes de carbohidratos altamente degradables en rumen, puede, en conjunto con el tiempo de acceso al pasto estrella africana, lograr un aumento en el consumo total de materia orgánica y así favorecer un suministro de energía total mayor en la ración sin disminuir el consumo voluntario de forraje y la digestión de la fibra (Villarreal et ál. 2006, Campabadal 1996). El valor nutricional del pasto estrella africana en Monteverde es limitante en energía, lo cual es característico de los pastos tropicales (NRC 2001, Sánchez y Soto 1999b), sin embargo, mediante la adecuada escogencia de suplementos se puede optimizar la utilización de los nutrientes a nivel ruminal sin que el consumo voluntario ni la digestión de la fibra se vean afectados, principalmente en animales de alto potencial genético (Villarreal et ál. 2006, Campabadal 1996). De acuerdo con Sánchez y Soto (1999b), la principal limitante de los pastos tropicales para la producción láctea es su contenido energético. Con el uso de los valores del NRC (2001), se puede estimar que con base en su contenido proteico promedio, el pasto estrella africana en Monteverde puede producir 2,07 $\mathrm{kg}$ de leche por $\mathrm{kg}$ de MS consumido (202,7 g PC.kg-1 MS/98 g PC), mientras que su contenido energético promedio permitiría lograr 1,69 $\mathrm{kg}$ de leche por $\mathrm{kg}$ de MS consumido (1,24 Mcal. $\left.\mathrm{kg}^{-1} \mathrm{MS} / 0,74 \mathrm{Mcal}\right)$. De acuerdo con lo anterior, el pasto estrella africana tiene un contenido proteico con alto potencial para la producción láctea que debe optimizarse por medio de una adecuada suplementación; asimismo las prácticas de manejo tendientes a incrementar la energía aprovechable del pasto pueden reportar incrementos en la productividad láctea de las fincas de la zona.

\section{CONCLUSIONES}

El pasto estrella africana en la zona de Monteverde mostró ser más suculento durante la época lluviosa. Los productores de ganado de leche deben asegurar un consumo de MS del pasto adecuado, bien sea con el manejo del pastoreo o con la suplementación de fuentes forrajeras adicionales como paca de heno, ensilajes y pastos de corte semi-deshidratados que permitan mantener una adecuada tasa de pasaje y retención en el rumen sin que el consumo voluntario de pasto en el potrero se vea afectado por efecto de sustitución. 
El contenido proteico del pasto estrella africana en Monteverde, aún durante la época seca, no es limitante para satisfacer los requerimientos del ganado lechero en la zona (NRC 2001). Por tanto, se debe realizar una adecuada escogencia de suplementos que permitan optimizar la producción de proteína microbial en el rumen en sincronía con los tiempos de acceso al pastoreo. A pesar de que el pasto estrella africana mostró variaciones significativas en su valor nutricional, se considera conveniente utilizar una única fórmula de alimento balanceado durante el año y realizar ajustes en la ración del concentrado, así como la utilización de suplementos que contengan carbohidratos fácilmente degradables en el rumen como la melaza de caña de azúcar, la pulpa de cítricos fresca o deshidratada y subproductos agrícolas de fácil acceso en la zona; para fomentar la producción de proteína microbial.

Las condiciones climatológicas de Monteverde permiten producir forraje durante todo el año, sin embargo el manejo del pastoreo en las fincas debe ajustarse durante las diferentes épocas del año de tal forma que la productividad y persistencia de las pasturas de estrella africana no sea afectada; principalmente en las fincas con influencia climática más directa de la región Atlántica del país.

\section{AGRADECIMIENTOS}

Los autores expresan su agradecimiento a los propietarios de las fincas donde se realizó esta investigación: Sr. Orlando Vargas, Sr. Jorge Donato, Sr. Ildefonso Picado y Sr. Esteban Jara por la colaboración brindada durante el desarrollo de la investigación.

\section{LITERATURA CITADA}

ALAYON J.A., RAMÍREZ-AVILÉS L., KU-VERA J.C. 1998. Intake, rumen digestion, digestibility and microbial nitrogen supply in sheep fed Cynodon nlemfuensis supplemented with Gliricidia sepium. Agroforestry Systems 41:115-126.
ALLEN M. 2000. Effects of Diet on Short-Term Regulation of Feed Intake by Lactating Dairy Cattle. Journal of Dairy Science 83:1598-1624.

ANDRADE M. 2006. Evaluación de técnicas de manejo para mejorar la utilización del pasto kikuyo (Pennisetum clandestinum Hochst. Ex Chiov) en la producción de ganado lechero en Costa Rica. Tesis de licenciatura, Universidad de Costa Rica, San José, Costa Rica $225 \mathrm{p}$.

ARROYO C., ROJAS A., ROSALES R. 2003. Urea o pollinaza como suplemento proteico para toretes consumiendo ensilaje de pulpa de pejibaye. Agronomía Costarricense 27(2):69-73.

ASSOCIATION OF OFFICIAL AGRICULTURAL CHEMISTS. 2000. Official methods of analysis. 17 ed. Washington, D.C. 2000 p.

ASSOCIATION OF OFFICIAL AGRICULTURAL CHEMISTS. 1990. Official methods of analysis. 15 ed. Washington, D.C. 1008 p.

CAMPABADAL C.H. 1996. Utilización de las grasas en la alimentación del ganado de leche. Nutrición Animal Tropical 3:99-110.

CRUZ M., SÁNCHEZ J.M. 2000. La Fibra en la alimentación del ganado lechero. Nutrición Animal Tropical 6:3974.

DZOWELA B.H., KUMWENDA M.S.L., MSISKA H.D.C., HODGES E.M., GRAY R.C. 1990. Seasonal Trends in Forage Dry Matter Production of Some Improved Pastures and Animal Performance in Relation to Chemical Composition in Malawi. Animal Feed Science and Technology 28:255-266.

ELIZALDE J.C., MERCHEN N.R., FAULKNER D.B. 1999. Fractionation of fiber and crude protein in fresh forages during the spring growth. Journal of Animal Science 77:476-484.

GONZÁLEZ N.V., BOSCHINI C.F. 1996. Comportamiento de la producción de leche en hatos Holstein y Jersey del Valle Central de Costa Rica. Nutrición Animal Tropical 3:43-59.

HILL G.M., GATES R.N., BURTON G.W. 1993. Forage Quality and Grazing Steer Performance from Tifton 85 and Tifton 78 Bermudagrass Pastures. Journal of Animal Science 71:3219-3225.

JOHNSON C.R., REILING B.A., MISLEVY P., HALL M.B. 2001. Effects of nitrogen fertilization and harvest date on yield, digestibility, fiber, and protein fractions of tropical grasses. Journal of Animal Science 79:2439-2448.

LASCANO C.E. 2002. Caracterización de las pasturas para maximizar producción animal. Archivos Latinoamericanos de Producción Animal 10(2):126132.

MANDEBVU P., WEST J.W., HILL G.M., GATES R.N., HATFIELD R.D., MULLINIX B.G., PARKS A.H., CAUDLE A.B. 1999. Caparison of Tifton 85 and Coastal Bermudagrasses for Yield, Nutrient Traits, 
Intake, and Digestion by Growing Beef Steers. Journal of Animal Science 77:1572-1586.

MISLEVY P. 2002. Stargrass. Florida Cooperative Extension Service, Institute of Food and Agricultural Sciences, University of Florida, Gainesville, USA. 4 p.

NATIONAL RESEARCH COUNCIL. 2001. Nutrient Requirements of Dairy Cattle. $7^{\text {th }}$ rev. Ed. Washington, D.C. National Academy Press. 381 p.

ORTIZ R.M.A., GALINA M.A., CARMONA M.M.A. 2002. Effect of a slow non-protein nitrogen ruminal supplementation on improvement of Cynodon nlemfuensis or Brachiaria brizanta utilization by Zebu steers. Livestock Production Science 78:125131.

PANT H.K., MISLEVY P., RECHCIGL J.E. 2004. Effect of Phosphorus and Potassium on Forage Nutritive Value and Quantity: Environmental Implications. Agronomy Journal 96:1299-1305.

PÉREZ J.P., ALARCÓN B.Z., MENDOZA G.D.M., BARCENA R.G., HERNÁNDEZ A.G., HERRERA J.G.H. 2001. Efecto de un banco de proteína de kudzu en la ganancia de peso de toretes en pastoreo de estrella africana. Técnica Pecuaria en México 39:39-52.

RETANA J. 2012. Climatología de la región de Monteverde, Puntarenas. Registro histórico 1956-2011. Instituto Meteorológico Nacional.

SALAZAR S. 2007. Disponibilidad de biomasa y valor nutricional del pasto estrella africana (Cynodon nlemfuensis) en el distrito de Quesada, cantón de San Carlos. Tesis de licenciatura. Universidad de Costa Rica, San José, Costa Rica 96 p.

SÁNCHEZ J.M., SOTO H. 1999a. Calidad nutricional de los forrajes de una zona con niveles medios de producción de leche, en el trópico húmedo del norte de Costa Rica. Agronomía Costarricense 23(2):165171.

SÁNCHEZ J.M., SOTO H. 1999b. Estimación de la calidad nutricional de los forrajes del cantón de San Carlos III. Energía para la producción de leche. Nutrición Animal Tropical 5:31-49.

SÁNCHEZ J.M., SOTO H. 1996. Estimación de la calidad nutricional de los forrajes del cantón de San Carlos
I. Materia seca y componentes celulares. Nutrición Animal Tropical 3:3-18.

SINCLAIR T.R., MISLEVY P., RAY J.D. 2001. Short photoperiod inhibits winter growth of subtropical grasses. Planta 213:488-491.

VAN SOEST P.J., ROBERTSON J.B. 1985. Analysis of forages and fibrous feeds. Cornell University. Ithaca, New York. 165 p.

VAN SOEST P.J., ROBERTSON J., LEWIS B. 1991. Methods for dietary fiber, neutral detergent fiber and nonstarch polysaccharides in relation to animal nutrition. Journal of Dairy Science 74:3586-3597.

VILLALOBOS L., ARCE J. 2013. Evaluación agronómica y nutricional del pasto estrella africana (Cynodon nlemfuensis) en la zona de Monteverde, Puntarenas, Costa Rica. I. Disponibilidad de biomasa y fenología. Agronomía Costarricense 37(1):91-101.

VILLALOBOS L. 2012. Fenología, producción y valor nutritivo del pasto alpiste (Phalaris arundinacea) en la zona alta lechera de Costa Rica. Agronomía Costarricense 36(1):25-37.

VILLALOBOS L., SÁNCHEZ J.M. 2010. Evaluación agronómica y nutricional del pasto ryegrass perenne tetraploide (Lolium perenne) producido en lecherías de las zonas altas de Costa Rica. II. Valor nutricional. Agronomía Costarricense 34(1):43-52.

VILLARREAL M., COCHRAN R.C., ROJAS A., MURILLO O., MUÑOZ H., POORE M. 2006. Effect of supplementation with pelleted citrus pulp on digestibility and intake in beef cattle fed a tropical grass-based diet (Cynodon nlemfuensis). Animal Feed Science and Technology 125:163-173.

WINGCHING R., CABALCETA G., ALVARADO A. 2009. Impacto del pastoreo con Ganado Holstein y Jersey sobre la densidad aparente de un andisol. Agronomía Mesosamericana 20(2):371-379.

WINGCHING R., PÉREZ R., SALAZAR E. 2008. Condiciones ambientales y producción de leche de un hato de ganado Jersey en el trópico húmedo: el caso del Módulo Lechero-SDA/UCR. Agronomía Costarricense 32(1):87-94.

Todos los derechos reservados. Universidad de Costa Rica. Este artículo se encuentra licenciado con Creative Commons Reconocimiento-NoComercial-SinObraDerivada 3.0 Costa Rica. Para mayor información escribir a rac.cia@ucr.ac.cr 
